\title{
Trends und Herausforderungen
}

\section{Fünf Handlungsempfehlungen für Telekommunikationsanbieter}

Gestern noch innovativ, morgen schon veraltet. Das gilt in Zeiten der Pandemie umso mehr - wirkt sie doch an vielen Stellen wie ein Brennglas. Gerade in der Telekommunikationsindustrie ist und bleibt die Disruptionsdynamik konstant hoch. Dabei wird deutlich: COVID-19 ist zwar herausfordernd für die Branche, kann Innovationen aber auch beschleunigen und die Wettbewerbsfähigkeit der Anbieter katalysieren - insbesondere im E-Commerce-Umfeld. Wo liegen die Chancen? Und worauf kommt es jetzt an?

Steven Bailey

Wirtschaftsinformatik \& Management 2021 • 13 (5): 384-389

https://doi.org/10.1365/s35764-021-00348-5

Angenommen: 8. Juni 2021

Online publiziert: 3. August 2021

๑) Springer Fachmedien Wiesbaden GmbH, ein Teil von Springer Nature 2021 
CSPs müssen in der Lage sein, ihr digitales Geschäft kontinuierlich anzupassen, um weiterhin wettbewerbsfähig zu bleiben. Das heißt: kommerzielle Angebote überdenken und neu gestalten, neue Techniken einsetzen, innovative Dienste in die Portfolios integrieren. Und verinnerlichen: „Experience is the new product!“ Dass der stetige Wandel das „New Normal" ist, hat spätestens seit der Pandemie die meisten Branchen erreicht. Für die Telekommunikationsindustrie lautet die Preisfrage: Wie können Communication Service Provider (CSPs) von dieser Entwicklung profitieren? Vor allem, da viele Leistungen wie etwa die reine Konnektivität immer stärker als Norm und Handelsware betrachtet werden, andere Akteure in die wertschöpfungsintensiven Segmente vorstoßen und der Verdrängungswettbewerb - häufig von den nichttraditionellen Akteuren ausgehend - zunimmt. Vor dem Hintergrund des allgegenwärtigen Ziels, Umsätze und Marktanteile zu steigern, haben viele CSPs erkannt: Es ist Zeit, ein echtes datengetriebenes „Echtzeitunternehmen“ zu werden. E-Commerce-Umsätze und Time-to-Market zählen dabei zu den entscheidenden Erfolgsfaktoren, die erodierende Qualität der Kundenkontakte und digitale Disruptoren zu den größten Herausforderungen. Eines wird hier schnell klar: Telekommunikationsunternehmen brauchen im ersten Schritt eine zukunftsfähige, auf Offenheit basierende technologische Basis, um ihre Daten gewinnbringend nutzen, Einnahmen steigern und neue, differenzierende Mehrwerte generieren zu können. Fünf Empfehlungen für „Telcos“, die halbherzige Projekte hinter sich lassen und ein digitales Framework für das E-Commerce-Umfeld von heute und morgen schaffen wollen.

\section{Den Kunden in den Mittelpunkt stellen - jetzt aber wirklich (eSIM lässt grüßen)}

Der äußerst wettbewerbsintensive Kampf um die Kunden läuft ja nicht erst seit gestern. Bedürfnisse und Wünsche von Kundengruppen und einzelnen Nutzern zu verstehen und einen guten Kontakt zur Kundenbasis zu halten, ist für Telekommunikationsanbieter daher mehr denn je das Gebot der Stunde. Aktuell erlebt die Branche eine rasante Veränderung des Kundenverhaltens, die neue organisatorische und technologische Strategien erfordert. Customer Journeys sind zunehmend fragmentiert, neue Touch Points entstehen, digitale Interaktionen und mobil dominieren. Wo vorher der menschliche Kundenkontakt entweder im Shop oder per Telefon ganz selbstverständlich war, bleibt oft nicht viel davon übrig.

Ein gutes Beispiel für Desintermediation: der von eSIMs ausgelöste Paradigmenwechsel und die Herausforderungen,

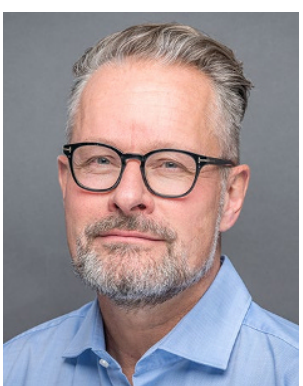

Steven Bailey $(\square)$

Steven Bailey verfügt über eine langjährige Expertise in der digitalen Transformation internationaler Unternehmen und der Entwicklung ihrer Business- und IT-Visionen. Als Chief Strategy Officer verantwortet er bei $A O E$ das Business Development und die Kundenberatung im Bereich Digitalisierungs- und Omnichannel E-Commerce-Strategien. steven.bailey@aoe.com

${ }^{1} \mathrm{AOE}$ GmbH, Wiesbaden, Deutschland

die damit einhergehen. Diese „embedded“ SIM-Karten sind fest in Geräte eingebaut und können softwareseitig mit den Profilen und Netzwerkinformationen unterschiedlicher CSPs aktiviert werden. Gerätehersteller oder andere Anbieter haben so die Möglichkeit, selbst ein Bündel aus Hardware und Kommunikationsdienstleistungen, die Konnektivität auf Abruf bieten, zu schnüren. Traditionelle CSPs können leicht umgangen werden, verlieren ganz automatisch den direkten Kundenkontakt und werden zu „White-Label“-Zulieferern. Womöglich werden sie hier auch die Teilnahme an weltweiten Allianzen suchen, um wieder selbst Kommunikationsdienstleitungen $\mathrm{zu}$ monetarisieren. Und - ebenso bedeutsam - Plattform Economy zu ihrem Vorteil einsetzen, indem sie gezielt und intelligent ihre Präsenz in sozialen Netzwerken aufbauen (Abb. 1).

Zusätzliche Erlösquellen könnten sich darüber hinaus in diesem Segment auftun, etwa Maschinen oder Haushaltsgeräte mit integrierter Mobilfunkkonnektivität. Ein gutes Beispiel hierfür ist Telefonicas M2M-Technologie, die beispielsweise von Nestlé als Basis für IoT für Kaffeemaschinen eingesetzt wird. In jedem Fall gilt es, sich zu positionieren und im Sinne des Kunden nach vorne zu denken. Gelebte Customer Centricity - nicht nur auf dem Papier. 


\section{$\mathrm{KI}$ und Machine Learning zur Kundenbindung einsetzen - mit Predictive Analytics anfangen}

Was wollen und brauchen Kunden denn nun ganz konkret in einer bestimmten Situation oder Interessenlage, zu einem bestimmten Zeitpunkt? Je relevanter und individueller die Informationen über neue Produkte, Services und Angebote sind, umso größer sind die Chancen, Kunden zu gewinnen und nachhaltig zu binden. Dies auch vor dem Hintergrund von Vergleichs- bzw. Opinion-Portalen und der öffentlich sichtbaren Bewertung durch Kunden. Voraussetzung ist ein zielgenaues Marketing, das auf bestimmte Kunden-Personas und in der jeweils notwendigen Granularität auf die Anforderungen der Kunden ausgerichtet ist. Durch entsprechende Kundenkanäle, das datenschutzkonforme Nutzen und Anreichern von Kundendaten in einem modernen CRM-System können schnell aufschlussreiche Einblicke gewonnen und für personalisierte Marketingkampagnen genutzt werden.

Der Umgang mit technischen Innovationen wie KI (künstliche Intelligenz) und Machine Learning wird hier für Telekommunikationsunternehmen zum wirtschaftlichen Erfolgsfaktor. Bereits im Einsatz befindet sich Predictive Analytics - eine Technik, die dabei hilft, interne und externe Daten zu analysieren, Muster zu erkennen und Trends zu antizipieren. Techniken und Herangehensweisen wie beispielsweise Regressionsanalyse, multivariate Statistik, Musterabgleich, prädiktive Modellierung und Vorhersage machen dies mög- lich. Enormes Potenzial verbirgt sich darüber hinaus in weiteren Einsatzbereichen von KI und Machine Learning. So können im Telco-Sektor künftig Szenarien unterstützt werden, die etwa der korrelationsbasierten Netzwerkanalyse bis hin zur Aufdeckung von Abrechnungsbetrug dienen (Abb. 2).

Intelligente und selbstlernende Systeme geben den Telcos nicht nur Werkzeuge an die Hand, um ihre Kunden besser zu verstehen und eine immer zielgenauere Kundenansprache in Echtzeit vorzunehmen. Sie ebnen auch den Weg für eine Endto-End-Digitalisierung ganzer Prozessketten. Also: Ran an KI und Machine Learning, nicht nur für inkrementelle Verbesserungen, sondern auch um neue Kundenerlebnisse zu ermöglichen.

\section{Bundling, 5G, loT, Cloud und mehr: Chancen sehen und neue Geschäftsmodelle denken}

Digitale Inhalte und CSPs - das ist nicht neu. So hat sich beispielsweise Viacom seit 1994 mit der Übernahme der Mehrheit von Paramount Pictures und später mit verschiedenen Akquisitionen wie CBS und Dreamworks Studios konsequent von einem CSP zu einem Medienkonzern entwickelt. Für eher traditionell aufgestellte CSPs wird digitaler Content nun ein immer wichtigeres Instrument zur Kundenbindung. Hierauf zahlt auch die Pandemie ein, in der die Verbraucher zu Hause bleiben und viel Zeit vor dem Bildschirm verbringen. Streaming steht dabei hoch im Kurs, während das lineare Fernse-

\section{Abb. 1 Plattform Economy}

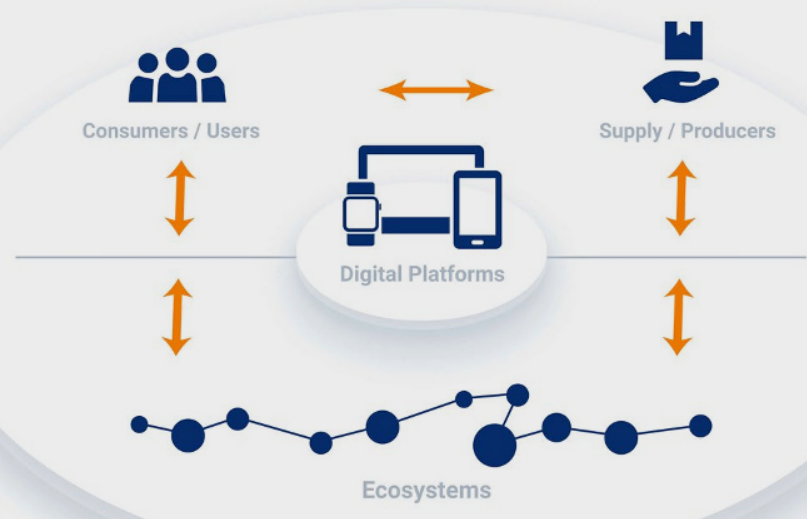


hen nicht dazugewinnt oder sogar zurückgeht. Partnerschaften mit Netflix, Amazon Prime oder Disney sind mittlerweile schon kein Differenzierungsmerkmal mehr, sondern eher ein Commodity-Produkt, das von den Kunden schlicht erwartet wird. Somit müssen CSPs eine aktivere Rolle im Markt für bestehende und künftige digitale Dienste spielen, um Marktanteile zu gewinnen oder auch nur zu erhalten. Dabei gibt es im Hintergrund unterschiedliche technische Möglichkeiten: von der Nutzung eines externen Dienstes unter eigenem Logo bis hin zu gemeinsamen Systemen.

Ein weiteres interessantes Bundling-Beispiel bahnt sich derzeit an: Manche CSPs erwägen, künftig auch die Dienstleistungen von Energieunternehmen anzubieten - und umgekehrt. Die Tarife berechnen sich ähnlich und basieren auf vergleichbaren Strukturen, das heißt der IT-Aufwand im Hintergrund könnte überschaubar umgewidmet werden.

Zwar wird 5G derzeit noch mehrheitlich wie schnelles $4 \mathrm{G}$ angesehen und behandelt, doch werden Telekommunikationsanbieter in den Geschäfts- und Unternehmensmärkten dadurch zunehmend in der Lage sein, bestehende Businessmodelle zu erweitern oder neue zu erschließen, zum Beispiel im Gesundheits- oder Bildungswesen oder beim autonomen Fahren. Niedrige Latenzzeiten von unter $1 \mathrm{~ms}$ und hohe und planbare Netzwerkbandbreiten bis zu $10 \mathrm{GBit} / \mathrm{s}$ machen es möglich. Auch im privaten Bereich öffnet 5G Türen für neue Formate, zum Beispiel Cloud Gaming via Google Stadia, GeForce Now, Vortex oder Magenta Gaming. Die bisherigen Netze mit geringerer Bandbreite können im Gegenzug künf-

\section{Zusammenfassung}

- Digitales Geschäft kontinuierlich anpassen

- Halbherzige Projekte hinter sich lassen

- Digitales Framework für das E-Commerce-Umfeld schaffen

tig für Datentransfers mit niedrigem Netzwerkprofil eingesetzt werden, etwa im Bereich IoT (Internet of Things).

Der Cloud-Markt wächst ebenfalls kräftig. Angetrieben wird dies zusätzlich durch die Pandemie und die zunehmende Arbeit im Homeoffice, wodurch sichere Speicher noch mehr an Bedeutung gewinnen. Der Marktanteil der US-Tech-Riesen Amazon, Microsoft und Google wird auf $60 \%$ geschätzt und es entsteht der Anschein, als hätten diese Anbieter den Cloud-Kuchen unter sich aufgeteilt. Hier bietet sich für CSPs dennoch nennenswertes Potenzial. Dies gilt für Telekommunikationsangebote, die mit den Cloud-Diensten verbundenen sind, wie für kundenorientierte Angebotsbündel (Telekommunikations-, Datendienste und IT-Systemintegration) oder für Mehrwertdienste wie Application Hosting, Sicherheitserweiterungen und Edge Computing. Hier stehen dann genauso eigene Services oder Partnerschaften mit etablierten Anbietern im Vordergrund - oder Kombinationen aus beidem.

Das Wichtigste, wenn es um neue Geschäftsmodelle und Erlösquellen geht: Keine Denkverbote!

\section{Abb. 2 Predictive Analytics}

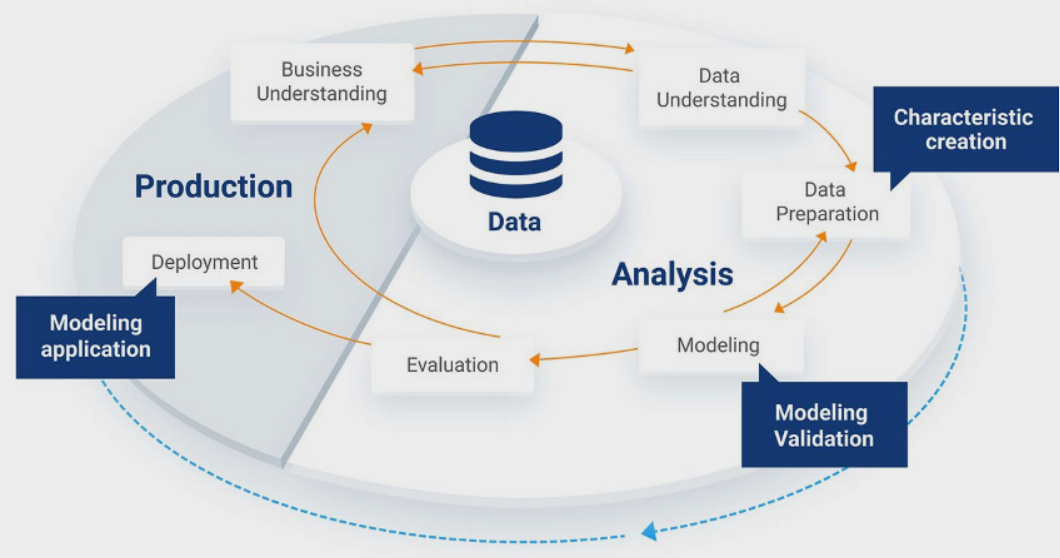




\section{Kernthesen}

- Transparenz statt Komplexität

- Modularität und Wiederverwendbarkeit

- Entwicklung von Composable-Architecture-Paradigmen

\section{Digitale Disruptoren entzaubern}

Neue Player am Markt erhöhen zusätzlich den Wettbewerbsdruck für Telekommunikationsanbieter. Während diese DigitalNative-Organisationen sämtliche Technologien beherrschen, tun sich traditionelle Telco-Anbieter oft schwer mit neuen Ansätzen wie Microservices-Architektur, ereignisgesteuerte Architektur oder programmierbare Infrastruktur. Ein Beispiel: Bisher ist es den meisten Telekommunikationsbetreibern trotz des großen Interesses nicht gelungen, eine nennenswerte Nachfrage über die reine Konnektivität hinaus für ihre IoT-Angebote zu generieren. Stattdessen sind es die nichtorthodoxen Marktteilnehmer, die den Markt geknackt haben. Neben günstiger Preispositionierung ist dies häufig auch auf einfache Transaktionsmodelle (direkter Onlinekauf) zurückzuführen. Zahlreiche
Anbieter aus diesem Segment verfügen überhaupt nicht über eigene Netze, es werden ausschließlich Produkte oder Dienstleistungen angeboten. Ein gutes Beispiel an dieser Stelle ist der Onlinedienst IFTTT (If This Then That). Sowohl Unternehmen als auch Endnutzer können über die Webseite Apps, Endgeräte oder Services via sogenannten Applets intelligent miteinander verknüpfen. Dies kann recht trivial sein, beispielsweise leuchtet jedes Mal eine grüne Lampe auf, wenn der Lieblings-Fußballklub ein Tor schießt, aber auch sehr komplexe Ereignisabläufe abbilden. Der IFTTT-Marktplatz umfasst heute bereits einige Zehntausend dieser Applets. Folglich hilft Telcos nur: eigene Toplösungen aufbauen oder einen erfolgreichen Disruptor akquirieren (oder beides) und so den raschen Markteintritt schaffen. Die Voraussetzung ist in jedem Fall ein offenes, flexibles Geschäftsmodell sowie ein Verständnis für den schmalen Grat zwischen Wettbewerb und Kooperation.

\section{Erst digitale Transformation, dann Strategie- wechsel? Die richtige Reihenfolge wählen - und dann Tempo machen}

Um wirklich liefern zu können, was der moderne und informierte Kunde verlangt, müssen Telekommunikationsun-

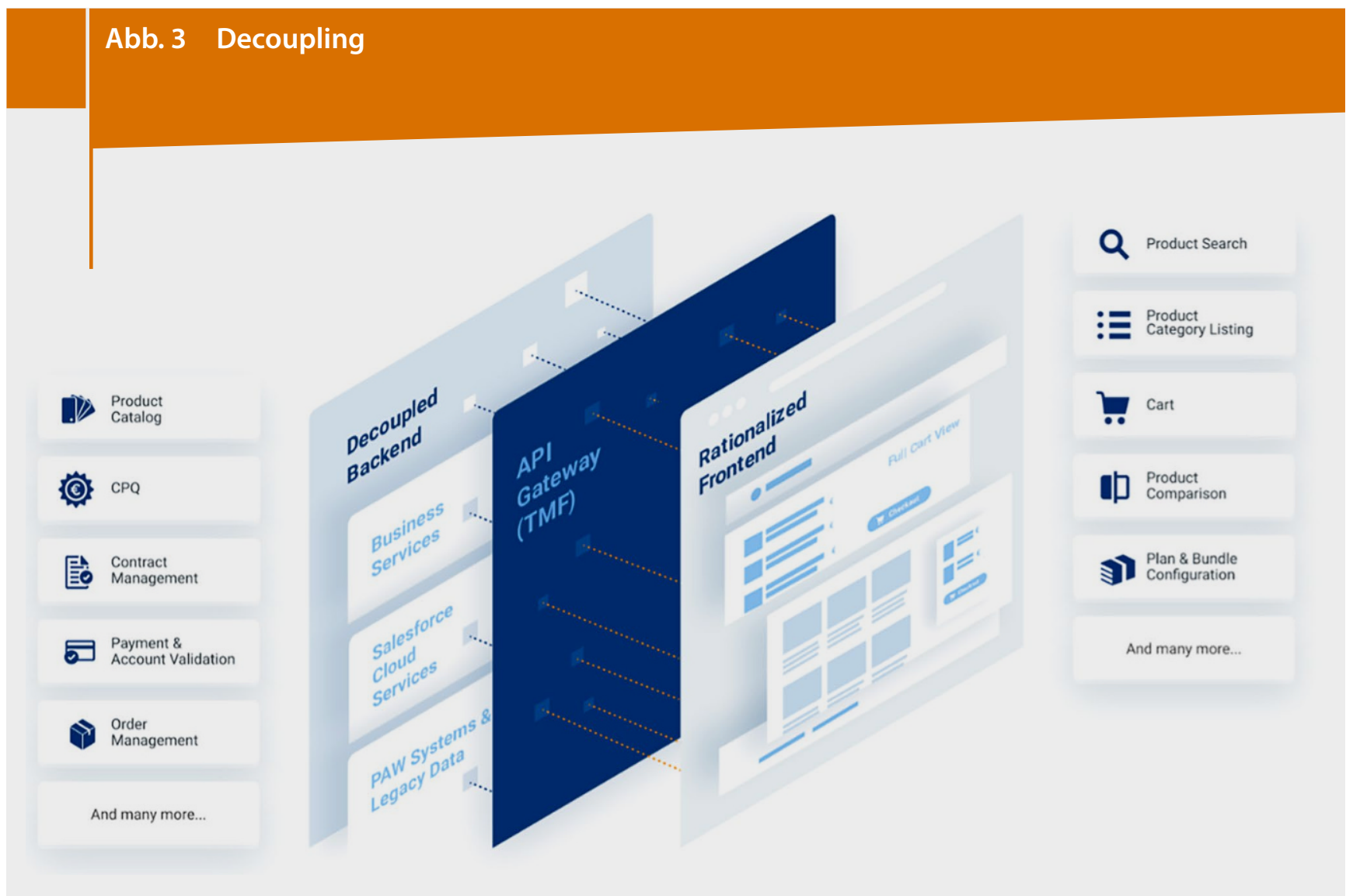


ternehmen zunächst den Weg in Richtung einer digitalen Transformationsstrategie einschlagen. Die anschließende Umsetzung dieser Digitalstrategie muss gar nicht gesamthaft erfolgen, besser in kleinen, bewältigbaren Schritten vorgehen und diese regelmäßig evaluieren. So kann man Zwischenergebnisse und Erkenntnisse gleich für alle weiteren Maßnahmen nutzen. Gleichzeitig behält man den Überblick und bleibt flexibel für Anpassungen an der ausgearbeiteten Strategie. Die Technologieplattformen, die bei traditionellen Telekommunikationsunternehmen heute im Einsatz sind, haben oft schon viele Jahre auf dem Buckel. Kein Wunder, dass die IT-Kosten aufgrund der Komplexität und der Redundanzen der Altsysteme viel zu hoch sind. Monolithische Architekturen bilden häufig das unflexible Rückgrat (Backend/ Legacy-System), über das die meisten Transaktionen abgewickelt werden (zum Beispiel Zahlungsverkehr, Order Management, Verfügbarkeitscheck etc.). In einem Umfeld, das auf agile Entwicklung und Microservices setzen muss, stehen sie der Digitalisierung im Weg. In diesen historisch gewachsenen Szenarien müssen sich die Geschäftsmodelle noch weitgehend nach den Möglichkeiten der IT richten - und nicht umgekehrt. Eine schlechte Nachricht für die Wettbewerbsfähigkeit, denn Erfolgsfaktoren wie Flexibilität und Time-toMarket können durch die starren Technologien und Prozesse kaum ermöglicht werden.

Dabei gibt es viele Möglichkeiten, Legacy-Anwendungen in moderne Architekturen zu integrieren. Der gängigste Ansatz ist die Verbindung über APIs und Konnektoren. In diesem Zusammenhang wird das sogenannte „Decoupling“, bei dem das kundenorientierte Frontend von der Backend-Schicht entkoppelt wird, zu einem zentralen Thema (Abb. 3).

Anbieter können auf diese Art personalisierte Kundenerlebnisse schaffen, die Freiheit zum Experimentieren gewinnen, ihre Agilität erhöhen und effizienter skalieren. Weitere Touch Points mit den Kunden können so jederzeit flexibel über die APIs in das Frontend eingebunden werden. Die Zielsetzung: sowohl erhebliche Kostensenkungen realisieren als auch eine Optimierung der wesentlichen KPIs Produktivität, Effizienz, Qualität und Time-to-Market.

\section{Handlungsempfehlungen}

- Den Kunden in den Mittelpunkt stellen

- Chancen sehen und neue Geschäftsmodelle denken

- Digitale Disruptoren entzaubern
Das Jahr 2020 hat uns gezeigt, wie schnell sich die Dinge ändern können und mit welcher Geschwindigkeit die digitale Transformation rast. Telekommunikationsunternehmen müssen sich eher heute als morgen zu digitalen Service-Providern und E-Commerce-Playern wandeln, um den sogenannten „Digital Dragons“ Paroli bieten zu können. Ein Begriff, den die Analysten von Gartner für Unternehmen geprägt haben, die bereits ein funktionierendes Online-Geschäftsmodell auf einem sehr hohen Skalenniveau haben, beispielswiese Amazon Web Services oder Alibaba.

Transparenz statt Komplexität, Modularität und Wiederverwendbarkeit mit Standards und offenen Schnittstellen sowie die Entwicklung von Composable-Architecture-Paradigmen - so kann digitale Transformation selbst im Eiltempo gelingen. Höchste Zeit für Telco-Anbieter, jetzt zu handeln. 